\title{
It Takes Two to Tango: Merging Science and Creativity to Support Continued Innovation in the IoT Domain
}

Helen Hasenfuss ${ }^{1,}{ }^{*}$, Muftah Fraifer ${ }^{1}$, Sameer Kharel $^{1}$, Asma Elmangoush ${ }^{2}$, Alan Ryan ${ }^{1}$, Walid Elgenaidi ${ }^{3}$

${ }^{1}$ CSIS-IDC, University of Limerick, V94 T9PX, Ireland

${ }^{2}$ The College of Industrial Technologies-CIT, Misurata, Libya

${ }^{3}$ Optical Fiber Sensors Research Center-OFSRC, University of Limerick, V94 T9PX, Ireland

\begin{tabular}{l} 
A R T I C L E I N F O \\
\hline Article history: \\
Received: 28 June, 2018 \\
Accepted: 03 September, 2018 \\
Online: 18 September, 2018 \\
\hline Keywords: \\
IoT \\
Technological innovation \\
Infrastructure \\
Cloud computing \\
Human behavior \\
UX
\end{tabular}

\begin{abstract}
A B S T R A C T
The Internet of Things (IoT) is a new concept that has a great appeal for researchers, businesses and the ordinary tech user. It presents new possibilities of connection between devices and people and it stimulates our need and desire to interact, to exchange ideas and to communicate with the surrounding environment. This paper briefly explores aspects of the IoT that may be important for future developments and focuses on the impact of creative methodologies, such as user centered design (UCD) in a smart parking, IoT-based system prototype. It aspires to provide an alternative perspective for an improved user experience $(U X)$ and aims to contribute to the discussion about the challenges, findings and perspectives when merging science and creativity to maintain continuous progress in the IoT domain.
\end{abstract}

\section{Introduction}

This paper is an extension of the article Look before you Leap presented in the conference IEEE 3rd international Forum on Research and Technologies for Society and Industry [1]. In that paper the complex system which was explored was a smart parking system through the application of the Internet of Things (IoT). It briefly discussed issues that relate to the IoT domain such as fundamental requirements, access technologies, security and privacy and data handling. The primary difference of approach in the development of this particular iteration of a smart parking system was the direct involvement of the users. The inclusion of users and/or the end users is a clearly defined methodology within design or human-computer interaction (HCI) disciplines, referred to as user centred design (UCD).

In order to preserve the quality and integrity of technological innovation it is important to combine methodologies from the science, technology, engineering and maths (STEM) disciplines and the Arts disciplines. A discussion of the methodological interplay between these diverse disciplines, as well as an attempt

\footnotetext{
*Helen Hasenfuss, University of Limerick, Helen.Hasenfuss@ul.ie
}

to expand on the challenges that emerge in the application of UCDbased methodologies into industry driven projects, are the two primary objectives of this paper. The IoT is a network of connections between digital and physical data / objects and providing a seamless user experience for IoT products is becoming an integral aspect of product design. Improved user experience (UX) is not always straightforward, as UX designers need to be knowledgeable about both the visible and invisible layers of IoT (e.g. visible layers: user interface, physical form factor and hardware spec; invisible layers: enabling architecture and technology backend, end user targets, etc.). The smart parking system that is discussed aims to highlight the impact of the involvement of the user in the design process. As IoT projects do not deal with singular issues of society (e.g. interface design, ergonomics, computing hardware, production quantity, etc.), it is necessary to shift some focus into developing multidisciplinary experts that can communicate between diverse disciplines (e.g. product design \& psychology) but that are also capable of translating between academic research and industry requirements. The growth and interest in the IoT is substantial as it is estimated that in 2020, 20.8 billion devices will be connected via the Internet, according to Gartner, Inc [1]. Approximately two billion people 


\section{H. Hasenfuss et al. / Advances in Science, Technology and Engineering Systems Journal Vol. 3, No. 5, 82-91 (2018)}

worldwide connect to the Internet in order to avail of the variety of services ranging from playing online games, social media, commerce, education, networking sites, entertainment and many more [2].

There is great potential for the concept of the IoT because it is expanding into and incorporating a variety of diverse research areas, see Figure 1. This expansion is advantageous because of the potential advancement of peripheral technologies, e.g., developing more efficient infrastructure to improve digital and physical resource management, energy sources for nanotechnology or advancing sensor technology.

Section two, the related work, will summarise the key issues that were discussed in the related conference paper [1]. These relate to the challenges, requirements, and applications that can influence the accessibility of the IoT, especially from a UX perspective. The continuous evolution and refinement of the IoT is reflective of how technology in the computer industry developed. For example, the physical hardware of the first computers dictated the design, layout and user interaction style of the IoT. Once technology became established and grounded, researchers began to consider the user and how they interacted and engaged with the technology. A natural progression from this consideration was for the user to be directly involved in the design process itself. Since the importance and value of the user is now established, the application of UCD methodologies alongside technology design processes ensures that adaptable and sophisticated IoT products / tech can be developed without needing to apply a singular approach to design.

The user and their experience are key elements in the development of the smart parking system [1]. An analysis of why it is important to consider the user in future technological is discussed in section three. The challenges of implementing more creative methodologies such as the user centred design, agile method into industry-based projects is illustrated and the value of their contribution is explored.

In the fourth section, the smart parking system that was developed in the Interaction design centre (IDC) in the University of Limerick (UL) between 2013 and 2017 will be discussed. The approach as to how the implementation of the UCD method influenced the development of the technical and economic aspects of the project are highlighted.

The fifth section is a discussion that briefly considers elements that affect IoT based projects but also analyses whether the recombination of STEM and Arts methodologies can make a difference in the approach to developing long term and lasting technological innovation. In an instant gratification society, endurance is becoming a rare characteristic even though it is still widely sought. The question of whether quantitative or qualitative research has the greater impact is explored from the perspective of science and artistic disciplines.

\section{Related Work}

It has become well known that technical challenges of IoT are diversified. In this section, we classify and identify some of these challenges.

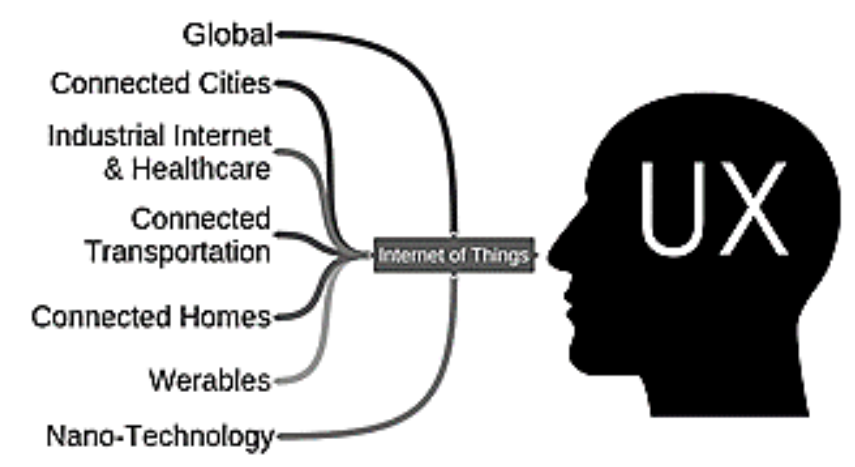

Figure 1. The influence of the IoT on local to global landscapes

\subsection{Security and Privacy}

One of the major concerns about IoT is security, as heterogeneous objects related to multiple domains could be connected to the Internet and communicate with various application services. Users worry that unauthorized third parties may be able to get access and/or control over their appliances and misuse data from the personal or Industrial applications [3]. Potentially, sensitive data will be roaming via the global Internet. Furthermore, most integrated components within an IoT system are characterized by low power and computation capabilities, and therefore cannot implement complex security mechanisms. IoT holds the potential of connecting everything and everyone, while not everyone should have access to everything.

\subsection{Access Technologies:}

Access control is necessary as well as data authentication in new communication technologies have been introduced for IoT with the objective of simplifying the deployments and gaining wider network coverage without compromising cost or power consumption. New technical solutions have been implemented that present an IP-based backhaul where the end-devices are provided with direct connectivity to a base station implementation a longrange wireless technology such as SIGFOX (SIFOX 2018) or short-range wireless technology such as Zigbee [4]. The use of the long- or short-range wireless technology depends on the application domain and the operational requirements. Table 1 summarizes the main characteristics of some other IP Protocols widely used in IoT projects. Many of these technologies are used in a variety of domains including home automation, sensor applications, and smart grids. Interested readers are referred to Naito's research for further discussion and insights of listed technologies for IoT [5].

\subsection{Compatibility and longevity}

The existing IoT deployments are growing in many different directions, with many different technologies competing to become the standard. Many Standard organizations have adopted a Representational State Transfer (REST) based architecture in their IoT framework such as ETSI and OneM2M, while few others have used the SOAP such as IEEE1888 [1,4]. In addition, more data protocols such as CoAP, XMPP, and MQTT are developed to address the requirements of integrating resource-constrained devices and supporting ubiquitous access. 
It can potentially cause complications and might involve the deployment of additional proxies and interworking software when connecting devices.

Table 1. IoT standard and Protocols

\begin{tabular}{|c|c|c|c|c|c|}
\hline Protocol & $\begin{array}{c}\text { Max } \\
\text { Range }\end{array}$ & $\begin{array}{c}\text { Max Data } \\
\text { Rate } \\
\text { (Kbps) }\end{array}$ & $\begin{array}{c}\text { Life of } \\
\text { Batteries }\end{array}$ & $\begin{array}{c}\text { Max } \\
\text { Num of } \\
\text { Devices / } \\
\text { base } \\
\text { station }\end{array}$ & Topology \\
\hline Sigfox & $50 \mathrm{Km}$ & 1 & 20 years & $\sim 1$ Mil & star \\
\hline Neul & $10 \mathrm{Km}$ & 100 & $10-15$ & $\mathrm{n} / \mathrm{a}$ & star \\
\hline \begin{tabular}{c} 
LoRaWAN \\
\hline Z-Wave
\end{tabular} & $15 \mathrm{Km}$ & 37.5 & 10 & $\sim 10 \mathrm{~K}$ & Tree \\
\hline $\begin{array}{c}\text { Zigbee } \\
\text { Bluetooth } \\
\text { low-Power }\end{array}$ & $100 \mathrm{~m}$ & 100 & Few years & 232 & Mesh \\
\hline 6LoWPAN & $200 \mathrm{~m}$ & $250 \mathrm{~K}$ & $\begin{array}{c}\text { Few } \\
\text { months }\end{array}$ & 100 & Mesh \\
\hline
\end{tabular}

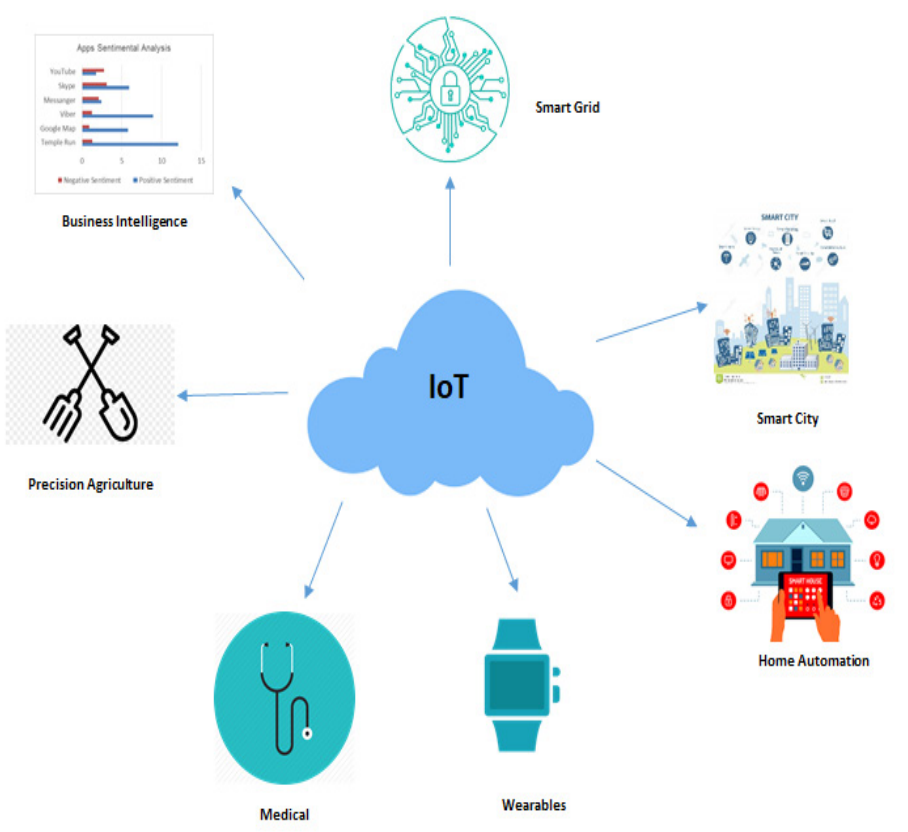

Figure 2. IoT fields

\subsection{Fundamental requirement for IoT}

IoT may also be a profitable venture for users in various businesses. It could potentially enable systems and smart manufacturing by connecting machines so that an industry can create networks along the entire life-time chain of a product or service $[1,6]$. The Telecommunication Sector of the International Telecommunication Union (ITU-T) has specified high-level requirements of IoT in Y.2060 recommendation (ITU-T 2012).

\subsection{Applications}

IoT applications are now starting to integrate various domains such as personal, home, enterprise, utilities and environment, as illustrated by Figure 2 .

Many services and applications have been developed, for example, in the medical domain with ubiquitous health care using sensors and body area networks to upload medical data to servers. Such home monitoring systems enable family members or community members (e.g. neighbourhood groups) to be notified about the condition of a person. This can potentially reduce the cost of health-care through pre-emptive action which can lead to an improvement in community awareness and care [7].

\section{User experience challenges}

IoT is growing every day and focus has been on technical aspects such as IoT infrastructure requirement, operating systems, security, power consumption, data, and storage. These technical aspects are relevant factors from an industrial perspective. However, involvement of users in any stage of development process has a positive impact on any product and improve the UX of the product. In an IoT ecosystem, different devices communicate with each other, possibly with different protocols. Different protocols and interfaces of devices have added complexity for users in its use in its ecosystem [8].

The tug of war between UX and user interfaces (UI) is an additional challenge to UX designers to provide seamless user experience without compromising security (Maiman 2015) [9]. Unification of interfaces to provide seamless experience is most challenging for UX designers in IoT. An interoperable design that can be easily adapted to the complexities of an IoT ecosystem is crucial to overcome this problem. Unification of interfaces across different appliances seems to be the biggest challenge for IoT user experience designers, as IoT products consist of different visible layers over a number of invisible layers, and designing a uniform experience requires much consideration (Janaway 2016, Staff 2016). While IoT UX can also be improved once IoT products are in market by getting feedback from users, changing physical design may not be straightforward due to its cost [9]. UX designers need to understand the different IoT invisible modules/layers that play a part in user interaction, like implementation of hardware, connectivity, data as IoT products are built on top of an IoT platform. Another challenge in interface design is that brands can have different requirements for the same software when used on different devices for example Android and iOS devices. Designing a UI for the IoT can be challenging since it, in many cases, is highly interconnected with other products, systems and services which affects the users' perceptions of their experiences.

The IoT can have a positive impact in the life of many citizens (users). It can be used to study human behaviour and understand how technologies are used in a daily life and this study can be useful for further IoT technology design. Involvement of the user via user centred design (UCD) in IoT design can't be ignored. The majority of IoT projects are still based in a lab setting, however ideally testing should be performed in real scenarios with real end users to find real problems [10]. 


\section{H. Hasenfuss et al. / Advances in Science, Technology and Engineering Systems Journal Vol. 3, No. 5, 82-91 (2018)}

\subsection{Activity Centred Design (ACD) vs User Centred Design (UCD)}

It is argued that the concept of UCD is not relevant in case of IoT. Cruickshank, L. and N. Trivedi suggest that the human should be seen as one of the smart objects in the IoT ecosystem and should fit into an activity centered design (ACD) approach. Cruickshank, L. and N. Trivedi argues that overall, the contribution of nonhuman elements is significantly higher than purely human elements in IoT systems. The discussion in academia relates to whether design should be able to keep up with emerging new agency rather than just try to satisfy users/customers [11].

In considering the ACD concept, we shouldn't forget that we are not trying to build robotic world but create robots for facilitating their (user/customer) daily life, maintaining privacy and without having tension between device and human. The results from Memedi's, research clearly shows that UCD plays important role in IoT. They found UCD as a helpful tool for identifying the requirement of the system user. For example, in a system developed for Parkinson's disease, patients it was found that $70 \%$ of users reacted positively to a IoT-based system prototype, (connected devices such as a wrist sensor, smartphone, bed sensor and an electronic dosing device). Positive impact of the system is due to including user in application/system development [12]. It was found that challenge of communication has been one of major component for hindering implementation of UX in the IoT domain. It was found that users are concerned about communication between device invisibly and users are unaware between information shared between it and different objects. It was also found users are interested in whether value is added to their life by connecting devices. Sometimes the complexity of IoT device configurations can also effect UX [13].

There are a variety of aspects that influence consumer acceptance of IoT systems and products. Factors that directly affect UX, range from tangible to nonphysical issues. For example, device memory and power: using many different IoT apps can be a drain on mobile devices or if it takes longer to complete a task using an IoT app than it would if it were done manually. Errors in IoT products can be detrimental to user experience, because the user can become irritated and generate more negative affect (see subsection 5.4). As a result, the user could simply switch to a similar product provided by an alternative company. Alternatively, qualities such as usefulness and trust have varying degrees of priority in relation to acceptance of IoT technology but are primarily dependant on the interaction and perception of the user.

Poor user experience for many IoT products is often due to ineffectual user-testing during the design process, leading to badly designed interfaces of applications and devices. For example, the smart heating system designed for supporting energy-saving lacked in all metrics used for its UX evaluation [14]. Users experienced difficulty accomplishing their tasks and the system also had inappropriate labelling [14]. Current trend of software development method has been developing rapidly, iteratively and incrementally and delivering modules of products to customer in short timeframes. The process is popularly known as "Agile".

\subsection{How practical is Agile in conjunction with UCD?}

The IDC in the University of Limerick, conducted interviews with 10 IT professionals working in software companies as UX experts, www.astesj.com designers and software developers from Ireland, Nepal and United State of America from August to October 2017. Every interviewee agreed that in some way they were following the Agile method and among different Agile methods "Scrum" is used in most cases. Agile is an umbrella term used for iterative and increment software development methodologies. Scrum is one of the sub-methods under Agile. In Scrum in every two to four weeks one complete module of the product is delivered to the customer which is known as a sprint.

From the above paragraphs it is clear that UCD should ideally be a mandatory concept in the development of user friendly and seamless UX in IoT devices. Brhel, M., et al., Butt, S. M. and S. M. Butt, Sohaib, O. and K. Khan have been writing about integrating the concept of UCD into the Agile development method [15-17]. A fundamental difference between agile development method and UCD is that the former focuses on the customer and the latter on the user. User and customer are almost the same, but every customer may not be an end user. For example, from a business perspective, everyone is a customer but, in our case, "user" refers to specific or targeted end users of a particular product, i.e. for a smart parking system based in UL - drivers who knew the campus grounds and administration were the appropriate users. To improve UX, suggestions have been made to use the Agile methodology to develop IoT devices in conjunction with UCD [18].

Regarding how often the software industry actually uses the concept of UCD in the development of new products, from the previously mentioned interviews of 10 IT specialists, we learned that incorporating UCD is not a straightforward process. The IT specialists agreed that implementation of UCD depends upon,

A) budget of the project,

B) requirement of customers and

C) that it is the responsible of the customer to know about their users. (In this case customer refers to an organization who enlists the services of a company that specialises in developing IoT products and systems.)

In [19], the paper proposes that usability testing is the least preferable testing method compared with unit, integration, system and acceptance testing as this testing are done by technical experts rather than involving end users. Mostly usability testing is conducted by software engineers rather than usability or UX experts. They indicate that customers give less priority to usability testing rather than acceptance testing, alpha and beta testing. Lack of usability is often attributed to the following aspects: $35 \%$ due to lack of time, $20 \%$ due to lack of training/knowledge, $15 \%$ lack of budget and rest for other reasons [19]. It state that "companies generally do not give priority to usability and UX evaluation" and including the user in software development is taken as a burden or delay in time and increased cost for relatively small benefit [19]. Different UCD tools have be implied in the Agile methodology for usability and UX. The usability tools vary slightly according to the discipline in which they are applied. For example, in requirements engineering, ethnographical observation, card sorting, personas, task scenarios, scenario and storyboards, and prototyping are the primary tools used to evaluate usability. In the design discipline: screen snapshots, product style guide, navigation maps are used. In the evaluation process, heuristic evaluation, cognitive 


\section{H. Hasenfuss et al. / Advances in Science, Technology and Engineering Systems Journal Vol. 3, No. 5, 82-91 (2018)}

walkthrough, thinking aloud, and lab usability testing are implemented. Then, an evaluation by experts, well-designed usability tests and analysis of installed systems are processes used as defined by UCD or have been slightly modified according to project demand [20].

The process of developing a smart parking system by including the relevant users or stakeholder and the positive outcome of the project highlight that participatory- and user centred design methodologies are invaluable for the overall design process but in particular the early stages of project development. This postulation is supported through diverse academic literature [10, 12]. Consistency, intuitive design and good, logical mapping are just some of the cornerstones for the successful uptake of any design as illustrated by Don Norman in his book "The Design of Everyday Things" [21]. Both lab and field testing need to be performed on IoT devices with users. Lab testing is helpful to improve the design of products and field testing is helpful to improve the user experience. Different user experience evaluation techniques, such as contextual laddering, day reconstruction, experience sampling and UX curves, can be applied as per requirements.

\subsection{Discussion of identified research gaps when applying UCD}

One of the basic steps in using the UCD method is to know everything related to the existing problems of the system expected to be designed. In order to build up this knowledge base, initial research should be carried out on other systems that have the same purpose. Whilst research indicates that there is a need for smart parking system solutions, there were no previous studies published in the literature relating to the assessment of these systems that also take into account the views of users. As well as this research indicated that each solution had different technological requirements depending on which factors received the highest priority, e.g. cost effective, integration possibilities, interface, etc. Each prototype system has advantages and disadvantages in terms of the following criteria:

- $\cos t$

- reliability

- scalability

- accuracy

- communication type

- circuit complexity

- reliability

- method of operation

- ease of installation

- usability of the system

There is an inadequate research base to fully inform how to design and evaluate smart parking systems for users.

\section{Challenges and findings from our perspective in the IoT domain}

UCD is a broad term to describe a design process based on the integration of potential users and since this era is witnessing a huge technological revolution of future networks depends on the connecting different objects from watches, cars, to satellites. There is no question about it: technology has revolutionized every part of our life nowadays as the Internet is likely to have a dramatic impact on our daily lives as it evolves into an essential part of many systems such as smart transportation, and smart parking etc.

The dialectical question that exists among those interested in the IoT domain remains: do we really need to link all things to each other? Because the different answers to the same question have advantages and disadvantages to the benefits of that linkage. However, the stakeholder is the only one to be able to estimate the extent to which he can benefit from the possibility of linking different things together to achieve the desired goals. Enabling technology from the things around us to be able to interact with each other and with the user, has many advantages and disadvantages that require careful consideration before any system is designed to deliver its services in a meaningful manner. We see an urgent need to have a harmony between merging science and creativity to support continued innovation in the IoT domain.

Certainly, exploiting the things around them and looking for alternative solutions to improve the level of services that users need is a great challenge, especially with regard to longevity and sustainability. This will create solutions for many dilemmas and problems that people face in general in various aspects of life.

\subsection{IoT based smart parking system}

Fraifer et al., designed a smart system that helps to find parking spaces at the University of Limerick. The difference here is that they incorporated the user into the design process in an early process of user-centered design [22]. Three groups of users were used to explore the problem of finding parking on an enclosed but spread-out area and to design a series prototypes to resolve, or alleviate, the issues. These issues include,

- reducing pollution by reducing the amount of time drivers spend looking for parking spaces,

- finding spaces hidden by larger cars,

- real-time update of free parking spaces,

- discerning peak times for searching, parking and when spaces are freed up, etc.

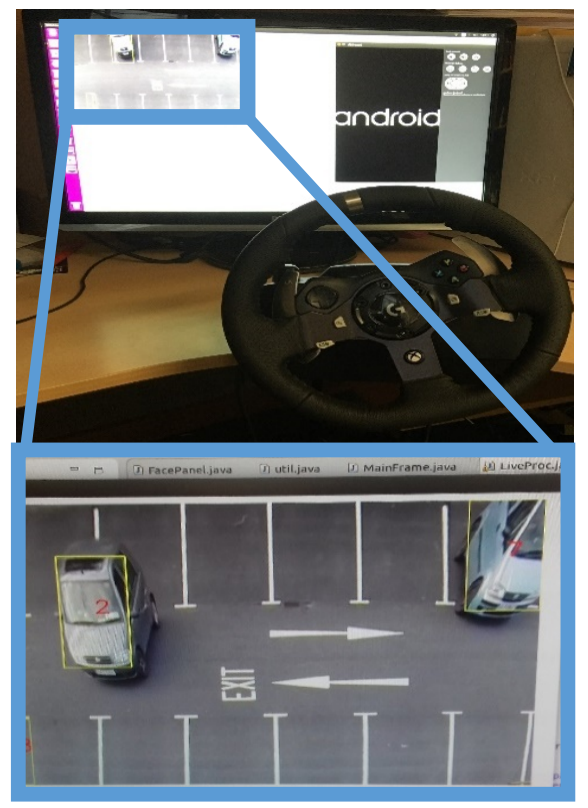

Figure 3. Illustrates how the CCTV footage was integrated in order create a search algorithm (left) and smart parking app (right of screen). 


\section{H. Hasenfuss et al. / Advances in Science, Technology and Engineering Systems Journal Vol. 3, No. 5, 82-91 (2018)}

The basis of the design implements CCTV which is often installed in any parking lot for the purpose of securing and preventing theft making it a viable economic solution. The user interacts with the system through a specific application in which they can see which places are vacant and where they can book a parking space [22], see Figure 3. The study has presented a design development of an application of IoT technologies. This has provided some findings that challenges the traditional approach to designing IoT based systems, e.g. how to make abstract technologies connective and interactable. Such findings helped to identify key areas of future research and intervention. They also support the argument for the need of new ideas and solutions that are mindful of the driver's requirements and how to improve the potential IoT smart parking system. Below is analysis data and finding discussion from two iterations of UCD approach.

\subsection{UCD methodology}

The UCD methodology frames the phases throughout a design and development of the prototype on gaining a deep understanding of those who will be using the system.

- Assigning the context of use: 12 members of the UL community participated in the smart parking project. These users were on campus on a daily basis and regularly experienced issues in finding parking. They include students, staff and frequent visitors

- Specify requirements: The users were interviewed and asked to complete a questionnaire regarding the primary issues they experienced in their normal parking routine. Within this phase it is possible to discern the major issues that should be addressed throughout the project but also what the users envisioned could potentially alleviate these issues.

- Create design solutions: Within this phase the majority of design work takes places and the actual product comes to fruition. For the smart parking project an app was developed that would enable users to find and reserve parking spaces cutting down on the time and fuel spent cruising around the large campus in order to find a parking space.

- Evaluate designs: This last phase is the crucial difference a UCD approach can make to product development. The evaluation procedure can be applied often throughout the design process and does not always require the full number of users. For example, once a low fidelity paper prototype was developed, that sufficiently conveyed the idea of the app, it was possible to evaluate it with some of the users. After this evaluation, the requirements, solution and evaluation phases can be continuously re-applied, until the final product becomes more refined and of a high-quality finish.

\subsection{Findings from designing an IoT based smart parking system at IDC}

The data analysis was based on qualitative findings from a humancomputer interaction perspective. From an user perspective, there is an inadequate research base to inform fully how to design and evaluate smart parking systems for users; therefore, this issue requires further investigation. A summary of the results and findings of research contributors from IDC are detailed in the following subsection 4.3.1 and 4.3.2.

\subsubsection{First iteration of $U C D$}

One of the most important benefits of this phase is the discovery of errors in the initial design of the system that would have led to failure if it had been left undetected. In addition, using different categories has main important benefits that has had beneficial results to redesign the prototype according to recommendations from users. The aim was to know the basic dilemma facing drivers while searching for a place to park. Other ideas relate to the development of solutions and ideas that may be part of the system.

\subsubsection{Second iteration of $U C D$}

The second iteration was used by a small group (focus group) consisting of 3 users. They were selected to perform a second session of UCD regarding the improvement of the system after the previous proposed modifications. This stage leads to the emergence of proposed system as a supposed final product. One of the findings in this stage and through the discussion with users, is that the system must include several things that contribute to solving the dilemma of finding a parking space. Below are the findings based on the data collection when applying the UCD approach. The initial model was an experiment in an indoor environment. The car park was designed using cardboard, where the camera was set up to face the simulated parking lot.

- The first idea was to draw circles with dark colours on every parking lot in order to facilitate the process of identifying whether the position is vacant or not. The system depended on computer vision, and it is easy to identify shapes by using the functions available in the OpenCV library.

- After presenting the model to the users, some of them made the comment that the system may not work in the event of snowfall as the circular shape is covered in winter by snow or autumn leaves. Users came up with the idea using actual cars, as that would be analogous to rectangles, using computer vision to detect if the parking lot is vacant.

- With regard to the designed mobile application, which is a fundamental element of the system, think-aloud sessions with users and two iterations of UCD enriched the appearance and usability of the model.

- The general tendency of participants was to make the application simple, but 9 users believed that the application would be more effective if it would show parking without activating reservation. They believed that using the application while driving may pose a risk to the driver. Enabling the driver to know the available vacant positions is in itself a good solution.

- To navigate directly to the vacant space to save time, fuel and mental effort, which is a basic goal of the system.

The primary concern of the 9 participants was that the system does not contain barriers to guarantee a parking space and gave the impression that the general system may take some time to function properly. Some users suggested activating the possibility of booking a specific space. In the cases where there is a fee for booking the space, the administration would be responsible for the space, should someone take it without a reservation. Developing scenarios often identifies important aspects of using a product in the real world that may not otherwise identified and considered in a lab setting, for example, the workflow being disrupted by a phone 


\section{H. Hasenfuss et al. / Advances in Science, Technology and Engineering Systems Journal Vol. 3, No. 5, 82-91 (2018)}

call. Scenarios are useful throughout the design process, particularly when developing task descriptions for usability testing.

\subsection{Findings from designing an IoT based smart parking system at IDC}

The primary findings from the smart parking system at the IDC relate to the infrastructure which the system uses and how the users felt about using the system. The infrastructures (Wi-Fi and CCTV cameras) which this system used enabled a smooth integration and reliable service. Internet access is widespread throughout the campus and CCTV is used for campus security. In general, the users were happy with the system and the app that was developed because it was easy and quick to use and did not impede their searching process. Advantages of this system are the ability to find available parking spots, especially when they are not visible from a driving position, and that the number and location of free spaces are updated in real time. Table 2 summarizes the most important aspects that were defined by users in the $2^{\text {nd }}$ round of evaluation [27].

Table 2. Findings from $2^{\text {nd }}$ iteration of evaluating the smart parking system.

\begin{tabular}{|c|c|c|}
\hline Source & $\begin{array}{l}\text { Needs/Requirements/Features/ } \\
\text { Solutions//Ideations }\end{array}$ & Findings \\
\hline Student & $\begin{array}{l}\text { Using Google Maps API library } \\
\text { services to determine the } \\
\text { destination accurately, especially } \\
\text { in large parking areas. } \\
\text { The possibility of seeing the } \\
\text { position of the car in a real way } \\
\text { by connecting the system to live } \\
\text { broadcast, perhaps through live } \\
\text { images. }\end{array}$ & $\begin{array}{l}\text { Providing a precise } \\
\text { positioning service such as } \\
\text { Google Maps will be } \\
\text { better for the user (user - } \\
\text { 01 Second session) } \\
\text { The availability of a } \\
\text { service enables the user to } \\
\text { see parking spaces in real } \\
\text { form in the form of images } \\
\text { that have a positive impact } \\
\text { on the user when he/she } \\
\text { arrives (user - 01) }\end{array}$ \\
\hline Visitor & $\begin{array}{l}\text { Create a database for users by } \\
\text { asking the same user to enter } \\
\text { his/her favourite times to take } \\
\text { advantage of the vacant places for } \\
\text { others if possible. }\end{array}$ & $\begin{array}{l}\text { The availability of } \\
\text { database users will help } \\
\text { administrators to provide } \\
\text { available vacant spaces } \\
\text { smoothly, (user - 02) }\end{array}$ \\
\hline Staff & $\begin{array}{l}\text { The possibility of sending short } \\
\text { messages or notifications when a } \\
\text { user leaves a place before the end } \\
\text { of the reservation. } \\
\text { Provide information to the user } \\
\text { about each position on the } \\
\text { application, for example a counter } \\
\text { in descending order to make the } \\
\text { rest of the users aware of the } \\
\text { nearest position of silent vacant }\end{array}$ & $\begin{array}{l}\text { Enable the users to add the } \\
\text { SMS of informing others } \\
\text { that user leaves the pre- } \\
\text { booked place before the } \\
\text { end of the reservation. } \\
\text { (user - 03) } \\
\text { The availability of a } \\
\text { digital counter in } \\
\text { descending order showing } \\
\text { the end of the booking } \\
\text { represents a beautiful } \\
\text { feature to remind the user, } \\
\text { (user - 03) }\end{array}$ \\
\hline
\end{tabular}

\section{Discussion}

One challenge of technological innovation is its uptake into the public domain. The flexibility and adaptability inherent in the IoT concepts presents a myriad of new opportunities to find eloquent solutions as technology becomes more sophisticated. However, user integration into the technology design process is still a major obstacle. After a company identifies the appropriate target user group, which in itself can be challenging, extracting the relevant user data can be a deterring factor for the majority of companies. Collecting user data is often time and resource consuming and it is necessary to translate the user's requirements and opinions into data that a company can use. As has been highlighted throughout this paper it is necessary to,

A) Engage the users from early on in the design and development process for any IoT potential products or services,

B) Adapt the existing business models to support cost-effective services, [1]

In addition to adapting existing models in industry, it is also necessary to consider the methodologies used in academic research. Whilst this concept is the primary focus in the discussion section, subsections 5.1 to 5.4 briefly summarise previously addressed issues [1] in order to provide context for the extended discussion on the merging of science and creativity to continuing progressing technological innovation. These issues are as follows:

- Business requirements

- Existing infrastructure

- Global connectivity and networking

- Emotion-driven design

\subsection{Business requirements}

The IoT refers to uniquely identifiable objects and their virtual representations in an Internet-like structure. Special requirements are needed in order to manage, secure and facilitate these embedded software assets. These systems are considered an industrial subset of the IoT, which may include billions of interconnected sensors, devices and systems, many of which will communicate without human involvement [1].

\subsection{Existing infrastructure}

The global and expanding nature of the IoT means that the viability of the network in its entirety is an important factor. For such a dynamic system to continuing growing and expanding it is necessary to consider how this viability can be maintained and is affected by the diverse number of variables of which it is comprised, i.e. the system's stability, the existing infrastructure and availability of resources (bandwidth, storage capacity, IP address space, etc.). Similar to any human endeavour having a good grounding or baseline is important, e.g. grammar enables a person to gain command of a language and to understand it. In relation to the IoT, since it is built upon and using the existing infrastructure of the Internet it is necessary to keep reviewing the efficiency of the technologies involved. For example, if an older infrastructure can no longer support new technologies because either conceptual or physical compatibility between technologies is no longer possible, too many resources may be required in order to maintain it. This action would shift the focus from development, i.e. looking forward, to maintenance, i.e. being tied to the present and past. It correlates with Mark Weiser's description that a "good tool is an invisible tool." [23]. For example, becoming conscious of a failing infrastructure (glitches, connectivity issues, slow connection, limited development opportunities, etc) detracts from an actual IoT device or system. A good tool ensures that the user's focus is entirely on the task. 


\section{H. Hasenfuss et al. / Advances in Science, Technology and Engineering Systems Journal Vol. 3, No. 5, 82-91 (2018)}

\subsection{Global Connectivity}

The IoT is a network of connected devices and a key issue of connectivity is compatibility. To accommodate the question of compatibility raises questions regarding the concept of standardisation. In a system that is inherently built to accommodate diversity, this approach appears to be contradictory. Even though standards can ease the element of interaction with technology (e.g. the classic floppy disk as the save icon), it is necessary to maintain a balance between system diversity and strength. For example, allowing systems and devices to be compatible with each other can create an IoT network that is stable in the event of infrastructure degradation or failure due to physical factors, (e.g. power outage, out of range, no connection, no storage, etc.) [24]. Allowing the method of connectivity to be diverse can ensure that the whole system is stronger against attack and more adaptable. The latter option is also more analogous to the method in which groups of people interact with each other, e.g. language dialects.

Considering the IoT development from human aspects, such as communication, growth, and development, the interdependent nature of this type of network or system becomes evident. The IoT aims to be integrated into an environment that is inhabited by social, living entities. As a result, logic and reason must interact with emotion and unpredictability, e.g. technical developments are directly influenced by user experiences and vice versa. The social and communicative aspects that are inherent in the IoT system creates spaces for large as well as small communities to work together in order to generate smaller networks that can eventually be integrated or expanded further. People can gain a sense of "socio-technical responsibility" (de Rosney 2014) and can break down knowledge barriers.

\subsection{Emotional design}

The social aspect mentioned in section 5.3 highlights an intriguing challenge particularly from a design perspective: the unpredictability of human nature. The ideas put forward in phenomenology illustrate how multifaceted a single experience can be. Aside from design considerations such as ergonomics, emotion and thereby experience generation and perception are critical elements in the design process. In the early years of computer development, technology defined user interaction styles. Nowadays the awareness of the importance of designing for an experience as well as for efficient technology is the progress of technological development that is embodied in the IoT. Previously a user had to adapt their behaviour according to the technology. As the focus in industry shifted from pure technology development to aesthetics and user experience it was possible to see an adaptation in the design process. Instead of primarily emphasising the engineering and scientific methodologies, it became necessary to consider socio- and design-based methodologies. A part of the user experience is the emotion involved in the interaction with technology [25]. Automated teller machines (ATMs) are often referenced as a user interface whereby clear mapping, ease of use, informative feedback and an aesthetic yet practical design are essential qualities. Studies illustrate that an interface embodying these characteristics ensures that users interact with more patience and tolerance and that they have a greater capacity for creative problem solving should an error occur. Overly-complicated and frustrating designs can evoke "negative affect". As a result, users tend not to engage long enough in order to find appropriate solutions thereby creating negative experiences [26]. It is important to note that negative affect can provide valuable insights regarding developing interfaces that may also need to be used in times of emergency, whereby stress, pressure, or panic can seriously hinder decision-making processes. Considering a user's emotions and experiences in the design process, provides a greater understanding of the effects of human emotional states which in turn can aid in the development of technology that behaves more intuitively.

\subsection{Closing the gap between logic and creativity}

Identifying the appropriate stake holders is a key element once a UCD approach is implemented. Targeting the right users will be most cost effective and provide valuable and accurate data. For example, testing a sophisticated gaming interface (e.g. joystick) on users who don't play computer games will not yield accurate useable data. Not engaging or interacting with the target group of end-users throughout the design process could be detrimental because it could result in a design or product that does not meet user requirements or is not commercially viable. A key component to $\mathrm{UCD}$, aside from the involvement of the user, is the continuous evaluation process with the user, of the design itself. The repeated evaluation ensures that the primary aims, and requirements of the user are always up to date.

Design based methodologies are steps towards bridging the gap between scientific procedures and practice based artistic explorations. Merging science and creativity to support continued innovation in the IoT domain reflects the incorporation of new ideas that emerge through research into practical applications in the STEM disciplines. With respect to long term innovation, an argument can be made for a more active application of the STEAM approach as opposed to a purely STEM orientated procedure (the A in STEAM representing Arts). The generation of new ideas in research reflects in part the creative element of the human psyche [26].

The inclusion of the procedures evident in an artistic methodology has the potential to enrich those of the more logic and reasonbased disciplines. It is evident in history that there was little differentiation made between the arts and sciences, however an intuitive method of discovering or validating certain truths is based primarily on deductive reasoning. This type of reasoning involves determining specific facts and narrowing the field of exploration, i.e. number of variables that affect the system, in order to ascertain specific and conclusive truths. An analogy can be drawn to top-down processing: a researcher may start with several questions but eventually ends with one singular focus. The STEM disciplines mainly embody this type of reasoning approach and has been invaluable in defining specific procedures that facilitate repeatability, consistency and transparency in good scientific research [26].

An issue with an approach that encourages an exclusive focus on singular elements of a system or product, is that the research scope can become stale, it can miss peripheral yet related subjects and can become too rigid and unforgiving. For these reasons it is important to consider the methodologies applied in the arts, which 


\section{H. Hasenfuss et al. / Advances in Science, Technology and Engineering Systems Journal Vol. 3, No. 5, 82-91 (2018)}

in contrast to scientific or engineering disciplines are primarily based on inductive reasoning. Inductive reasoning embodies a bottom-up approach to research, i.e. it is the process whereby knowledge is acquired and constructed in order to generate questions that are further explored in the deductive process. An output of inductive inference are the probabilities of certain truths. For example, conclusions can be drawn logically from the knowledge acquired but it does not necessarily make accommodations if the knowledge base is inaccurate or incorrect. Therefore, the truths that emerge from inductive inference are a source of ideas and inspiration that can enrich the disciplines in which deductive reasoning is favoured [26].

The arts are primarily based on creativity - the ability to work with loose boundary conditions, the unknown or the unpredictable, to be able to see connections between diverse subject matter and to view errors as opportunities and not always as dead-ends. Similar to the sciences, a pure focus within the arts, e.g. dance, poetry, drawing, enabled discipline-specific methodologies to emerge (e.g. social art, practice-based methodologies). However, it is important to recognise that these methodologies in isolation are as self-limiting as those in the STEM disciplines.

The difference between logic and creative based disciplines is also reflected in the form of quantitative and qualitative output. Independently, each type of output has its value, but they also represent the different perspectives through which data can be interpreted. Therefore, it is not necessarily possible to declare that one form of output is better than the other, just as there is no one main research methodology that stands above others, but it is important for researchers to remain aware of these perspectives when using data and to apply a degree of objectivity.

In relation to long term technological innovation, a degree of time and energy must be invested into the early stages of technological development. In the generation of questions, it is important to draw conclusions that support a high probability of truth before the deductive approach is applied. Achieving this state indicates that the research must be thorough and accurate. The following example highlights the number of steps involved in taking an idea through the UCD process to create a high-fidelity prototype that is ready for industrial or public implementation. The even distribution of energy throughout these processes ensures that the end result is of good, reliable and repeatable quality.

- Idea generation through brainstorming with design team and ideally with relevant users

- Sorting of ideas into relevancy, possible pitfalls, new ideas, do's and don'ts

- Research one or two specific ideas and translate from idea into tangible concept

- Create a low fidelity prototype

- Run another brainstorming session with the prototype or run individual interviews

- Re-sort new contributions and research design and product elements further (e.g. material, platforms, hardware, etc.)

- Adjust and adapt the prototype to incorporate the feedback

- Run another evaluation session with the relevant users
- After the comments and feedback have been incorporated again it is possible to develop the prototype further.

- Create a high-fidelity prototype

- Implement the prototype and get real world data on interaction, glitches, successes, improvements, etc.

- Refine the prototype accordingly

- Run final tests with the prototype and evaluate the system via interviews with users

- At this point the prototype is thoroughly tested and can be moved on to the industrial phase whereby it could potentially be implemented beyond the test area.

The user centered design approach is one method by which an even distribution of energy and resources can be implemented into industry-based projects. Even though this process can initially take more time and resources, the results usually yield more accurate market research, public uptake and better quality of outputs, i.e. short-term versus long-term gain. The smart parking project described in this paper is an example of how important it is to consider the logical and creative aspects of the human psyche in the future development of technology.

The merging of logic and creativity advocates a flexible and adaptable approach to research. Whilst it is necessary to continue having singular-subject experts, to support technological innovation it is also necessary to train multidisciplinary experts. The latter type of expert and / or researcher develops the skill to communicate and translate between two or more diverse disciplines (e.g. biology, 3D printing and modelling). An advantage of this skill is the ability to alter perspective. It allows researchers to make connections between subjects or problems, etc, more quickly and they can bridge the gap between deductive and inductive inference.

\section{Conclusion}

The application and integration of design methodologies is a step towards integrating creative processes into scientific disciplines. Drawing out the skills in order to generate new ideas is vital in the enrichment of research scope. It is clear that the process of inductive inference (idea generation) and deductive inference (translating ideas into reality) is continuous and circular, as one phase feeds into the other. For this cyclical process to be successful it is necessary to find a balance in the application of creative and logical methodologies. In contrast focusing on either extreme (i.e. purely logic-based or creative-based methodologies) enables researchers to explore boundaries and limits of a specific discipline [26]. The peripheral topics that have been mentioned in this paper (e.g. business objectives, security, privacy, connection, etc) are not a conclusive list but demonstrate the range of interdependencies in a domain as versatile as the IoT. Even though the topics may not be directly involved, their influence can have interesting effects and outcomes on both the system in question and the related users. It is a constant interplay between variables that are known to the designer and variables that emerge as a result of implementation and real-life interaction. A chance always exists that designs may be used, not for what they were originally intended, but possibly for more creative or destructive purposes. It is another compelling reason for including users in 


\section{H. Hasenfuss et al. / Advances in Science, Technology and Engineering Systems Journal Vol. 3, No. 5, 82-91 (2018)}

the product development process as early as possible because even though it may initially cost more with respect to resources and time, the long-term benefits are evident in the quality and efficiency of products produced.

The smart parking system detailed in this paper explores the relation between the involvement of the user and the development of the technology. The results from this project are congruent with research studies on system development and usability, to facilitate user acceptance and efficiency, accuracy, and satisfaction. Because the users were involved through all four phases of the design process, their needs, suggestions, and preferences were continuously incorporated into the design and evaluation of an IoT based smart parking prototype. The UCD methodology transforms users into active contributors to the design process and has provided improved UX in each iteration. It enables the final product to be tailored directly around users' needs rather than providing superfluous functions that disrupt the design.

\section{Acknowledgments}

We would like to acknowledge the support of the Ministry of High Education Scientific Research-Tripoli, Erasmus Mundus LEADERS and Interaction Design Centre (IDC) in CSIS department at University of Limerick for their funding of the project.

\section{References}

[1] M. Fraifer, S. Kharel, H. Hasenfuss, A. Elmangoush, A. Ryan, W. Elgenaidi, and M. Fernström, "Look before you leap: exploring the challenges of technology and user experience in the internet of things" In Research and Technologies for Society and Industry (RTSI), 2017 IEEE 3rd International Forum on, 1-6, IEEE, Modena, Italy, 2017. https://doi.org/ 10.1109/RTSI.2017.8065920

[2] D. Miorandi, S. Sicari, F. De Pellegrini, and I. Chlamtac, "Internet of things: Vision, applications and research challenges" Ad hoc networks, 10(7), 14971516, 2012, https://doi.org/10.1016/j.adhoc.2012.02.016

[3] A. Grau, "Can you trust your fridge?" IEEE Spectrum, 52(3), 50-56, 2015. https://doi.org/10.1109/MSPEC.2015.7049440

[4] M. Tariq, Z. Zhou, J. Wu, M. Macuha, and T. Sato, "Smart grid standards for home and building automation" in 2012 IEEE International Conference on Power System Technology (POWERCON), pp. 1-6, Auckland, New Zealand, 2012. https://doi.org/10.1109/PowerCon.2012.6401448

[5] K. Naito, "A Survey on the Internet-of-Things: Standards, Challenges and Future Prospects" J. Inf. Process., vol. 25, pp. 23-31, 2017. https://doi.org/10.2197/ipsjjip.25.23

[6] D. Uckelmann, M. Harrison, F. Michahelles, "An Architectural Approach Towards the Future Internet of Things" In: Uckelmann D., Harrison M., Michahelles F. (eds) Architecting the Internet of Things. Springer, Berlin, Heidelberg, 2011. https://doi.org/10.1007/978-3-642-19157-2_1

[7] J. Gubbi, R. Buyya, S. Marusic, and M. Palaniswami, "Internet of Things (IoT): A vision, architectural elements, and future directions" Future generation computer systems, 29(7), 1645-1660, 2013 https://doi.org/10.1016/j.future.2013.01.010

[8] M. Dixit, J. Kumar, and R. Kumar, "Internet of things and its challenges" in 2015 International Conference on Green Computing and Internet of Things (ICGCIoT), 810-814, IEEE, Noida, India, 2015.https://doi.org/10.1109/ICGCIoT.2015.7380574.

[9] J. Bergman, T. Olsson, I. Johansson, and K. Rassmus-Gröhn, “An exploratory study on how Internet of Things developing companies handle User Experience Requirements" in International Working Conference on Requirements Engineering: Foundation for Software Quality, 20-36,
Springer, Cham, Utrecht, The Netherlands, 2018. https://doi.org/10.1007/978-3-319-77243-1_2

[10] M. Nati, A. Gluhak, H. Abangar, and W. Headley, "Smartcampus: A usercentric testbed for internet of things experimentation" In Wireless Personal Multimedia Communications (WPMC), 16th International Symposium on, 1-6, IEEE, Atlantic City, NJ, USA 2013. https://ieeexplore.ieee.org/stamp/stamp.jsp?tp=\&arnumber $=6618632$

[11] L. Cruickshank, and N. Trivedi, "Beyond Human-Centred Design: Supporting a New Materiality in the Internet of Things, or How to Design When a Toaster is One of Your Users" The Design Journal, 20(5), 561-576, 2017. https://doi.org/10.1080/14606925.2017.1349381

[12] M. Memedi, G. Tshering, M. Fogelberg, I. Jusufi, E. Kolkowska, and G. Klein, "An Interface for IoT: Feeding Back Health-Related Data to Parkinson's Disease Patients" Journal of Sensor and Actuator Networks, 7(1),14, 2018 https://doi.org/10.3390/jsan7010014

[13] J. Bergman, and I. Johansson, "The user experience perspective of Internet of Things development," Master thesis, Lund University, 2017.

[14] S. Wall, and F. Healy, Usability testing of smarter heating controls. A report to the Department for Energy and Climate Change. Amberlight. DECC, London,

2013https://assets.publishing.service.gov.uk/government/uploads/system/u ploads/attachment_data/file/266220/usability_testing_smarter_heating_con trols.pdf

[15] M. Brhel, H. Meth, A. Maedche, and K. Werder, "Exploring principles of user-centered agile software development: A literature review" Information and Software Technology, 61, 163-181, 2015. https://doi.org/10.1016/j.infsof.2015.01.004

[16] S.M. Butt, and S.M. Butt, "Usability Evaluation Method for Agile Software Development" International Journal of Software Engineering and Computer Systems, 1(1), 29-40, 2015. http://dx.doi.org/10.15282/ijsecs.1.2015.3.0003

[17] O. Sohaib, and K. Khan, "Integrating usability engineering and agile software development: A literature review" In Computer design and applications (ICCDA), 2010 international conference on (Vol. 2, pp. V2-32). IEEE, Qinhuangdao, China, 2010. https://doi.org/10.1109/ICCDA.2010.5540916

[18] X. Larrucea, A. Combelles, J. Favaro, and K. Taneja, K., "Software engineering for the internet of things" IEEE Software, (1), 24-28, 2017. https://doi.org/10.1109/MS.2017.28

[19] M.K Larusdottir, E.R. Bjarnadottir , J. Gulliksen, "The Focus on Usability in Testing Practices in Industry. In: Forbrig P., Paternó F., Mark Pejtersen A.” Human-Computer Interaction, vol 332. Springer, Berlin, Heidelberg https://doi.org/10.1007/978-3-642-15231-3_11

[20] D.A.Magües, J.W. Castro, and S.T. Acuna, "HCI usability techniques in agile development" In Automatica (ICA-ACCA), IEEE International Conference,pp. 1-7, IEEE, Curico, Chile, 2016. https://doi.org/ 10.1109/ICAACCA.2016.7778513

[21] D. Norman, The design of everyday things: Revised and expanded edition, Constellation, 2013.

[22] M. Fraifer, H. Hasenfuss, M. Fernström, "Taking Away the Green ScreenA Brief Discussion of a Multidisciplinary Approach to IoT via, Smart Parking and Interaction Design" In Smart Trends in Systems, Security and Sustainability, 33-45, Springer, Singapore, 2018. https://doi.org/10.1007/978-981-10-6916-1_4

[23] M.Weiser, "The world is not a desktop", interactions, 1(1), 7-8, 1994. https://doi.org/ 10.1145/174800.174801

[24] J.O Kephart, and.M. Chess, "The vision of autonomic computing" Computer, (1), 41-50, 2003. https://doi.org/ 10.1109/MC.2003.1160055

[25] D. A. Norman, Emotional Design: Why We Love (or Hate) Everyday Things, Basic Civitas Books, 2004.

[26] H. Hasenfuss, "A design exploration of an agent template for multiagent systems (MAS) for shape shifting tangible user interfaces," $\mathrm{PhD}$ Thesis, University of Limerick, 2018.

[27] M. Fraifer, "A design exploration of an IoT based smart parking system with stakeholders using User-Centred Design" unpublished $\mathrm{PhD}$ Thesis, University of Limerick, 2018 